\title{
Critical Casimir Interactions and Percolation: the quantitative description of critical fluctuations.
}

\author{
Oleg A. Vasilyev \\ Max-Planck-Institut für Intelligente Systeme, \\ Heisenbergstraße 3, D-70569 Stuttgart, Germany and \\ IV. Institut für Theoretische Physik, Universität Stuttgart, \\ Pfaffenwaldring 57, D-70569 Stuttgart, Germany
}

\begin{abstract}
Casimir forces in a critical media are produced by the spatial suppression of order parameter fluctuations. In this paper we address the question how fluctuations of a critical media relate the magnitude of critical Casimir interactions. Namely, for the Ising model we express the potential of critical Casimir interactions in terms of Fortuin-Kasteleyn site-bond correlated percolation clusters. These clusters are quantitative representation of fluctuations in a media. New Monte Carlo method for the computation of the Casimir force potential which is based on this relation is proposed. We verify this method by computation of Casimir interactions between two disks for 2D Ising model. The new method is also applied to the investigation of non-additivity of the critical Casimir potential. The non-additive contribution to three-particles interaction is computed as a function of the temperature and the separation between disks. The benefit of the proposed method is that it let us to compute the multi-particle interaction explicitly.
\end{abstract}

PACS numbers: 05.70.Jk, 64.60.Ak, 05.10.Ln 


\section{INTRODUCTION}

The critical behavior in the vicinity of the second order phase transition point is characterized by large scale fluctuations of the order parameter. The appearance of forces due to the spatial confinement of fluctuations in the vicinity of the critical point has been predicted by Fisher and de Gennes [1]. This phenomenon in a critical media is now known as the Critical Casimir (CC) effect [2-4]. One can observe the appearance of CC forces in a critical binary mixture near its consolute point [5]. Two colloidal particles in a fluctuating media exhibit attraction. The observation of colloidal particles aggregation in the critical binary mixture has been described in [6]. The interaction between a colloidal particle and a planar substrate has been measured directly my means of Total Internal Reflection Microscopy [7, 8]. Critical depletion in colloidal suspensions has been studied in many experiments [9-13] (see Ref. [14] for review). Recently, the non-additivity of CC interactions between three colloidal particles has been studied experimentally [15].

The analytical computation of CC forces is a difficult problem. A critical binary mixture belongs to the universality class of the Ising model. Therefore one can use numerical simulations of the Ising model to extract information about $\mathrm{CC}$ interactions for particular geometry and boundary conditions. The CC forces and its scaling functions for 3D Ising universality class with and without the bulk field for the film geometry and various boundary conditions have been studied numerically [16-21]. CC force between a spherical particle and a plane for the 3D Ising universality class has been studied in Ref. [22]. The numerical algorithm for the computation of CC interactions between two particles in the presence of the negative bulk field for 3D Ising model has been proposed in [23] and the interaction of a particle with two walls has been studied in [24].

Numerical methods also are used for the computation of critical interactions within mean field (MF) universality class. The CC force between two colloidal particles in 3D has been first studied using the conformal transformation in Refs. [25, 26]. MF interactions between an elliptic particle and a wall [27] and between two colloidal particles in the presence of the bulk ordering field [28] have been studied. Non-additive interactions for MF universality class have been investigated for plane-particle-particle [29] and three-particles [30] geometries.

Experimentally has been shown, that in a lipid membrane consisting of two different types of lipids the second order phase transition may occur [31, 32]. Such transition has 
been observed in a membrane forming plasma vesicles [33]. These experimental observations demonstrate the request for studying of the critical behavior of the 2D Ising model to which universality class they belongs. For 2D Ising model for the stripe geometry the CC force may be computed analytically [34-37]. Results for CC force between two disks for the 2D Ising model with the bulk field have been obtained via Derjuaguin approximation [38]. The alternative method for the computation of the $\mathrm{CC}$ interaction between a mobile disk and a wall has been recently proposed [39]. The interaction between inclusions in a critical 2D membrane has been studied in [40] by using the Bennett method. The torque, acting on a needle near a wall has been studied in Ref. [41]. The phase diagram of ternary solventsolvent-colloid mixture represented by 2D Ising model with disk-like particles has been investigated by using grand-canonical insertion technique [42]. Recently, the fluctuation induced Casimir interactions in colloidal suspensions at the critical point in 2D system has been studied by geometric cluster algorithm [43]. This algorithm capable to provide moving the particles and mixing the medium. Then two-particle and multi-particle interactions are extracted from particle distribution functions. For 2D Ising model at criticality one can use the power of conformal transformations for analytical evaluation of CC interactions between inclusions [33, 43]. Recently, conformal invariance has been used for the investigation of interactions between rod-like particles [44].

In this article we propose the short and elegant expression for $\mathrm{CC}$ interactions between objects based on the of critical percolation clusters. The introduced method is based on counting of a number of percolation clusters "touching" immersed objects. The paper is organized as follows: in the next section we express the interaction potential of CC force in terms of clusters of Fortuin-Kasteleyn site-bond percolation. We verify the numerical method by computing $\mathrm{CC}$ interactions between two disks and comparing with results of another numerical approach. Then the proposed method is applied to the computation of the non-additive interactions between three particles as a function of the inverse temperature. We and with a conclusions. 


\section{CASIMIR INTERACTION EXPRESSED VIA CRITICAL PERCOLATION CLUSTERS}

Let us consider the Ising model on a simple square lattice with periodic boundary conditions. All distances are measured in lattice units. The classical spin $\sigma_{i}= \pm 1$ is located in a site $i$ of the lattice. The inverse temperature is $\beta=1 /\left(k_{\mathrm{B}} T\right)$. The standard Hamiltonian of a bulk system (no restrictions for a spin directions) for a spin configuration $\{\sigma\}$ reads

$$
\mathcal{H}_{\mathrm{b}}(\{\sigma\})=-J \sum_{\langle i j\rangle} \sigma_{i} \sigma_{j},
$$

where $J=1$ is the interaction constant, the sum $\langle i j\rangle$ is taken over all pairs of neighbor spins. The partition function of the model is given by the sum over the total set $\langle\sigma\rangle$ of all spin configurations $Z_{\mathrm{b}}=\sum_{\langle\sigma\rangle} \mathrm{e}^{-\beta \mathcal{H}_{\mathrm{b}}(\{\sigma\})}$. The corresponding free energy of the bulk system is $F_{\mathrm{b}}(\beta)=-\frac{1}{\beta} \ln \left[Z_{\mathrm{b}}(\beta)\right]$. One can rewrite the partition function in terms of the FortuinKasteleyn correlated site-bond percolation [45] (reviews on percolation theory are Refs. [4648]). For every bond between two spins $\sigma_{i}$ and $\sigma_{j}$ we introduce the bond variable $n_{i j}$, which can take values 0 (open) and 1 (closed). We introduce the probability $p=1-\mathrm{e}^{-2 \beta}$ for a bond between two parallel $\sigma_{i}=\sigma_{j}$ spins to be closed $n_{i j}=1$. In our case of homogeneous interactions with the fixed constant $J=1$ the value of this probability $p$ does not depend on spin indexes $i j$. The probability for a bond between parallel spins to be open $n_{i j}=0$ is $1-p=\mathrm{e}^{-2 \beta}$ (the concept of open and closed bonds between parallel spins has been proposed by Coniglio and Klein [49]). A bond between two antiparallel $\sigma_{i}=-\sigma_{j}$ spins is always open $n_{i j}=0$. We denote $\{n\}$ the particular configuration of bond variables. The standard expression for the partition function may be rewritten as the sum over all set $\langle n\rangle$ of configurations of open and closed bonds

$$
\begin{gathered}
Z_{\mathrm{b}}(\beta)=\sum_{\langle\sigma\rangle} \mathrm{e}^{-\beta \mathcal{H}_{\mathrm{b}}(\{\sigma\})}=\sum_{\langle\sigma\rangle} \prod_{\langle i j\rangle} \mathrm{e}^{\beta \sigma_{i} \sigma_{j}}= \\
=\mathrm{e}^{\beta N_{b}} \sum_{\langle\sigma\rangle} \sum_{\langle n\rangle} \prod_{\langle i j\rangle}\left[(1-p) \delta_{n_{i j}, 0}+p \delta_{n_{i j}, 1} \delta_{\sigma_{i}, \sigma_{j}}\right]= \\
=\mathrm{e}^{\beta N_{b}} \sum_{\langle n\rangle}\left[p^{n_{c}(\{n\})}(1-p)^{N_{b}-n_{c}(\{n\})} 2^{c(\{n\})}\right],
\end{gathered}
$$

where $N_{b}$ is the total number of bonds in the system, $n_{c}(\{n\})$ is the number of closed bonds and $c(\{n\})$ is the number of cluster of connected spins in the configuration $\{n\}, 2=q$ is the number of spin states for the Ising model. The set $\langle n\rangle$ consists of $2^{N_{b}}$ different bond configurations. Clusters of parallel spins, connected by closed bonds are called "physical" clusters 

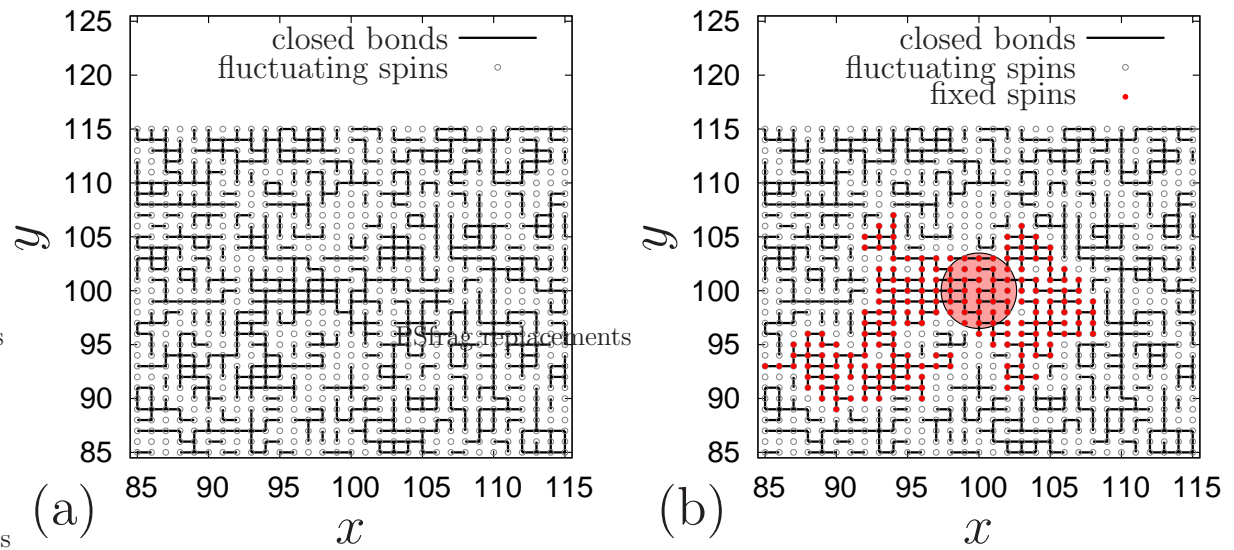

FIG. 1: (Color online) (a) Clusters of spins, connected by closed bonds. Each cluster can take either -1 or +1 orientations, these fluctuating spins are denoted by empty circles. (b) The same configuration of clusters with the inserted disk of the radius $R=2.5$ with the center at the point $(100,100)$. All clusters which contain spins within the circle should be fixed +1 . Fixed spins of these clusters are denoted by filled circles.

(contrary to "geometrical" clusters of parallel spins). Detailed derivation of the partition function and expressions of physical quantities like energy, specific heat, magnetization, and magnetic succeptibility in terms of physical clusters have been provided by De Meo, Heermann and Binder [50]. The Fortuin-Kasteleyn correlated site-bond percolation is the basis of the Swendsen-Wang numerical cluster algorithm for spin models [51]. We write eq.(2) in the form of the Ising model (e.g., $\sigma_{i}= \pm 1, q=2$ is the number of spin states). It is also possible to rewrite it for $q$-state Potts model $\left(\sigma_{i}=0,1, \ldots, q\right)$ with an arbitrary value of $q$. It means, that this approach generalizes CC interactions for the Potts model.

Let us clarify the definition of a cluster of connected spins. Two spins $\sigma_{i}$ and $\sigma_{j}$ are connected if the bond between these spins is closed $n_{i j}=1$, otherwise these spins are not connected. The set of all spins, connected between each other by closed bonds is called the cluster. In accordance with the definition of closed bonds, all spins in a given cluster are parallel. But not every pair of parallel neighbor spins belong to the same cluster. Let us note, that in the last line of eq. (2) the sum is taken only over the set $\langle n\rangle$ of all possible configurations of open and closed bonds (totally $2^{N_{b}}$ configurations). At the same time for every particular configuration $\{n\}$ the contribution from all possible -1 and +1 cluster 
configurations is given by the term $q^{c(\{n\})}=2^{c(\{n\})}$ which counts the number of clusters $c(\{n\})$ for this configuration. The example of such bond configuration on the square lattice is given in Fig. 1(a) where closed bonds are shown by solid lines and open bonds are not indicated. Directions of all spins in Fig 1(a) are not specified because each cluster can take both possible directions -1 and +1 . Let us now consider the system with immersed particle (or particles). In terms of the Ising model it means, that all spins of such particle are fixed $[22,23,39,43]$. In Fig. 1(b) the example of the disk of the radius $R=2.5$ with the center located in the point $(100,100)$ is demonstrated. We denote $\{$ col $\}$ the set of all fixed spins in our colloidal particle(s), in the current example $\sigma_{k}=+1$ for spins with coordinates $\left(x_{k}-100\right)^{2}+\left(y_{k}-100\right)^{2} \leq R^{2}$. The partition function $Z_{c}$ of the system with immersed colloidal particle(s) may be expressed via the Hamiltonian of a bulk system $\mathcal{H}_{\mathrm{b}}$ with the application of the constraint $\delta_{\sigma_{k}, 1}$ to all fixed spins. On the other hand, this partition function may be expressed via the third line of eq. (2) if we take into account, that all clusters $c_{c}$ which contain spins of the colloidal particle can not fluctuate. The partition function of the system with immersed particle(s) is expressed as

$$
\begin{gathered}
Z_{c}(\beta)=\sum_{\langle\sigma\rangle} \prod_{k \in\{\mathrm{col}\}} \delta_{\sigma_{k}, 1} \mathrm{e}^{-\beta \mathcal{H}_{\mathrm{b}}(\{\sigma\})}= \\
=\mathrm{e}^{\beta N_{b}} \sum_{\langle n\rangle}\left[p^{n_{c}(\{n\})}(1-p)^{N_{b}-n_{c}(\{n\})} 2^{c(\{n\})-c_{c}(\{n\})}\right] .
\end{gathered}
$$

The only difference from the partition function of the bulk system without particle(s) $Z_{\mathrm{b}}$ in eq. (2) is the term $2^{-c_{c}(\{n\})}$. This term reflects the fact, that all $c_{c}$ clusters of the current configuration $\{n\}$, which contain spins of colloidal particle, are fixed and do not contribute to the partition function. The free energy of a system with a particle $(\mathrm{s})$ is $F_{c}(\beta)=-\frac{1}{\beta} \ln \left[Z_{c}(\beta)\right]$. Therefore, we can expressed the free energy difference (in $k_{\mathrm{B}} T$ units) of the insertion of particle(s) as

$$
\beta\left[F_{c}(\beta)-F_{\mathrm{b}}(\beta)\right]=-\ln \left[\frac{Z_{c}(\beta)}{Z_{\mathrm{b}}(\beta)}\right]=-\ln \left\langle 2^{-c_{c}}\right\rangle_{\beta},
$$

where $\left\langle 2^{-c_{c}}\right\rangle_{\beta}$ is 2 to the power $-c_{c}$ where $c_{c}$ is the number of clusters, touching the immersed object computed as a thermal average $\langle\ldots\rangle_{\beta}$ at the inverse temperature $\beta$ with respect to the bulk (empty) system. The proposed method can also be applied to systems with various types of boundary conditions and the presence of the bulk field. Let us note, that we do not specify how many particles are immersed into a bulk system. It means, that the simple expression eq. (4) may be applied to computation of CC interactions for various geometries: 
particle-particle, multi-particles, wall-particle, etc. Moreover, this numerical method can be also applied to an arbitrary spatial dimension and for $q \neq 2$ Potts model.

\section{MODEL DESCRIPTION AND NUMERICAL VERIFICATION}

Let us demonstrate the application of the proposed method to the computation of CC interactions between two disks in 2D Ising system. This example has a practical interest, because it describes the interaction between protein inclusions in a lipid membrane at critical concentration of membrane components [31-33, 38, 42, 43].

We consider the 2D Ising model on a square lattice with the periodic Boundary Conditions (BC). All distances are measured in lattice units, the system size is $200 \times 200$. For the

reference bulk system with the free energy $F_{\mathrm{b}}(\beta)$ in each site of the lattice the fluctuating spin $\sigma_{i}= \pm 1$ is located. For the system with immersed particles with the free energy $F_{c}(\beta, D)$ two disks (disk 1 and disk 2) of the half integer radius $R=3.5$ are immersed in the system at the distance $D$ - see Fig. 2(a). Spins in these disks are fixed to be +1 . This
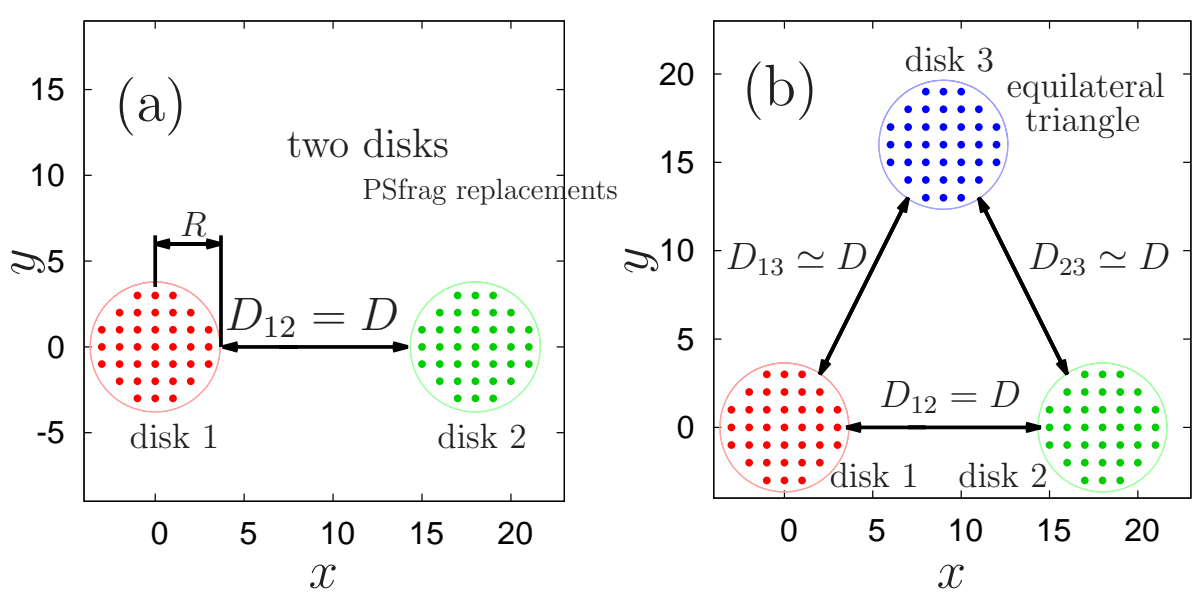

FIG. 2: (Color online) The system geometry for: (a) Two disks of the radius $R=3.5$ at the separation $D_{12}=D$ for the numerical verification of the algorithm; (b) Equilateral triangle of disks at separations $D_{12}=D, D_{13}=D_{23} \simeq D$ for the studying of non-additive interactions.

choice corresponds to the symmetry-breaking BC with completely ordered surface and it is usually denoted as $(++)$ BC (see [19] for details). Let us denote $c_{12}(D)$ the number of all clusters, which contain spins of two disks at the distance $D$. We denote the insertion free 
energy difference for two disks

$$
U_{\mathrm{ins}}^{12}(\beta, D)=\beta\left[F_{c}(\beta, D)-F_{\mathrm{b}}(\beta)\right]=-\ln \left\langle 2^{-c_{12}(D)}\right\rangle_{\beta}
$$

which depends on the distance between disks $D$ and is expressed via the logarithm of the average value of 2 to the power of $-c_{12}(D)$ in accordance with eq.(4). The insertion energy itself does not provide the information about disks interaction because it is defined up to a certain constant. Let us take as a normalization constant the insertion energy $U_{\text {ins }}^{12}\left(\beta, D_{\max }\right)$ which corresponds to the maximal possible separation $D_{\max }$ between particles for the finite system with periodic BC. In our case $D_{\max }=L-2 R$ where $L=200$ is the system size. We introduce the $\mathrm{CC}$ interaction potential

$$
\begin{gathered}
U_{12}(\beta, D)=U_{\text {ins }}^{12}(\beta, D)-U_{\text {ins }}^{12}\left(\beta, D_{\max }\right)= \\
=\ln \left\langle 2^{-c_{12}\left(D_{\max }\right)}\right\rangle_{\beta}-\ln \left\langle 2^{-c_{12}(D)}\right\rangle_{\beta},
\end{gathered}
$$

which derivative with respect to the distance provides the Casimir force $f_{\text {Cas }}=-\partial U_{12} / \partial D$ between two disks.

As a first step we numerically verify the algorithm by computing the interaction potential $U_{12}(\beta, D)$ between disk 1 and disk 2 in accordance with eq. (6). We use the hybrid Monte Carlo (MC) algorithm [52]. Each step consists of one update in accordance with SwendsenWang algorithm [51] (which is efficient in the vicinity of the critical point) followed by $L^{2} / 5$ attempts of Metropolis spin updates [53] at random positions (which is efficient out of criticality). The averaging is performed over $8 \times 10^{8} \mathrm{MC}$ steps splitted on 10 series for the evaluation of the numerical inaccuracy. Let us note, that in accordance with our algorithm we can simultaneously perform computations for a set of disks separations $D$. In Fig. 3 we plot by symbols the interaction potential $U_{12}$ between two disks as a function of the inverse temperature $\beta$ for various separations $D=1,3,5,7,11,19$. In the same figure we also plot by lines the potential computed by alternative reference method. The reference method (ref.) is based on the numerical integration of local magnetization over the locally applied field $[23,54]$. We observe the perfect agreement between results computed in two different ways. It confirms eq. (6) and verifies the numerical code which is used for simulations. The position of the critical value of the inverse temperature $\beta_{c}=\ln (\sqrt{2}+1) / 2$ is denoted by the vertical dashed line. The maximum of the attractive interaction is slightly shifted to high-temperature region as it is typical for $(++)$ BC [19]. The proposed method can 
be straightforwardly expanded for systems with bulk and surface fields and systems with several values of the interaction constants.

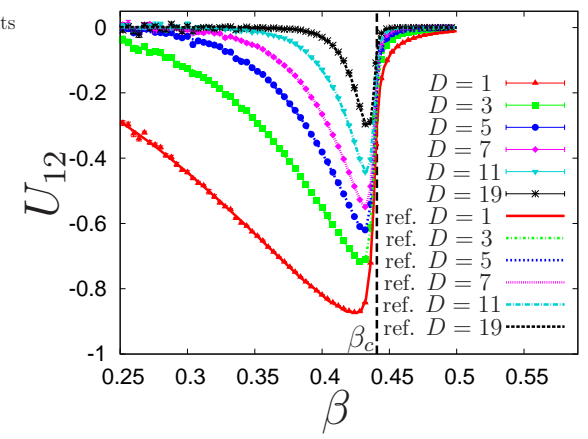

FIG. 3: (Color online) Numerical verification of CC interaction potential $U_{12}$ as a function of the inverse temperature $\beta$ between two disks at distances $D=1,3,5,7,11,19$ computed via percolation clusters (symbols) and by using the reference method [23] (lines).

\section{NON-ADDITIVITY OF THREE PARTICLE INTERACTIONS}

Let us now study the non-additivity of interaction in three-particles system with the geometry of an equilateral triangle. This geometry is shown in Fig. 2(b) and it has been used in experiment [15] and in the investigation of non-additivity for MF universality class [30]. Now we add the third disk 3 at the separation $D_{13}$ from the first disk and at the separation $D_{23}$ from the second disk. The separations selected to be almost equal on the lattice $D_{13}=$ $D_{23} \simeq D_{12} \equiv D$ Later on for the equilateral triangle we select the odd distance $D$ so the projection of the center of the third disk on $x$-axis is located exactly between centers of disk 1 and disk 2 .

We denote $c_{12}, c_{13}, c_{23}$, and $c_{123}$ the number of percolation clusters, which contain spins of disks 1 and 2, disks 1 and 3, disks 2 and 3, and all disks 1,2,3, respectively. The potential of three particle interaction is expressed as

$$
U_{123}(\beta, D)=\ln \left\langle 2^{-c_{123}\left(D_{\max }\right)}\right\rangle_{\beta}-\ln \left\langle 2^{-c_{123}(D)}\right\rangle_{\beta}
$$

We also introduce the sum of pair interactions $U_{123}^{s}=U_{12}+U_{13}+U_{23}$. For the additive potential we expect $U_{123}=U_{123}^{s}$. In Fig. 4(a) we plot $U_{123}$ as a function of the inverse 

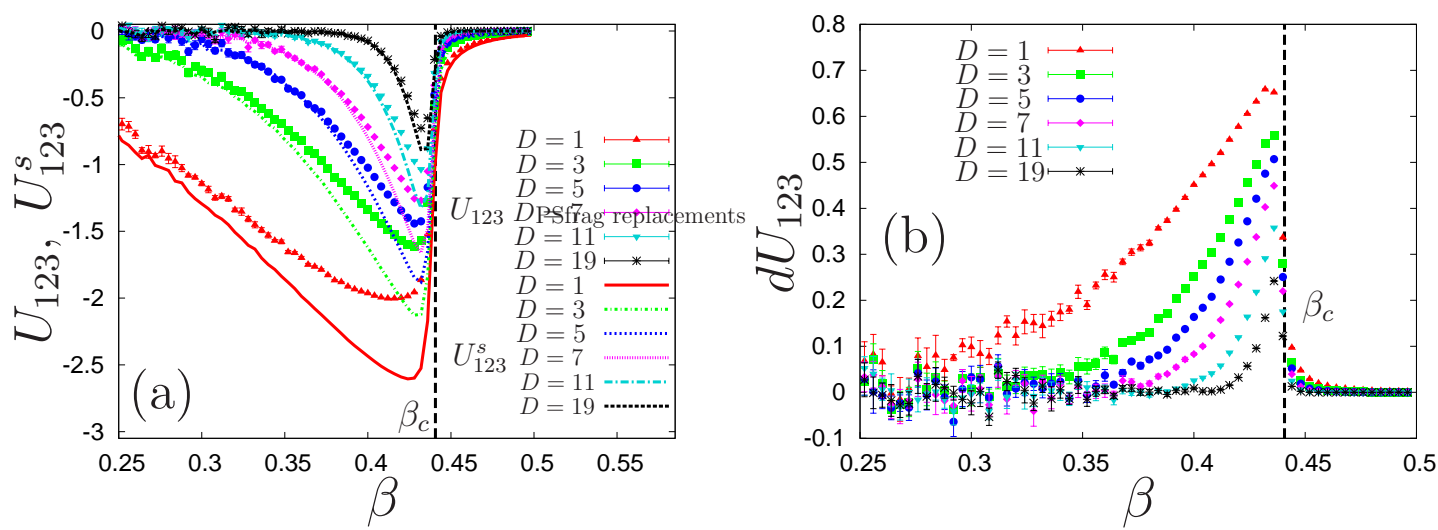

FIG. 4: (Color online) (a) Multiparticle CC interaction potential $U_{123}$ (symbols) between three disks located in vertices of the equilateral triangle with side of length $D=1,3,5,7,11,19$ as a function of the inverse temperature $\beta$ in comparison with the sum of three pair potentials $U_{123}^{s}=$ $U_{12}+U_{13}+U_{23}$ (lines); (b) Excess non-additive contribution to $\mathrm{CC}$ interaction potential $d U_{123}=$ $U_{123}-U_{123}^{s}$ as a function of the inverse temperature $\beta$ for three disks at same separations.
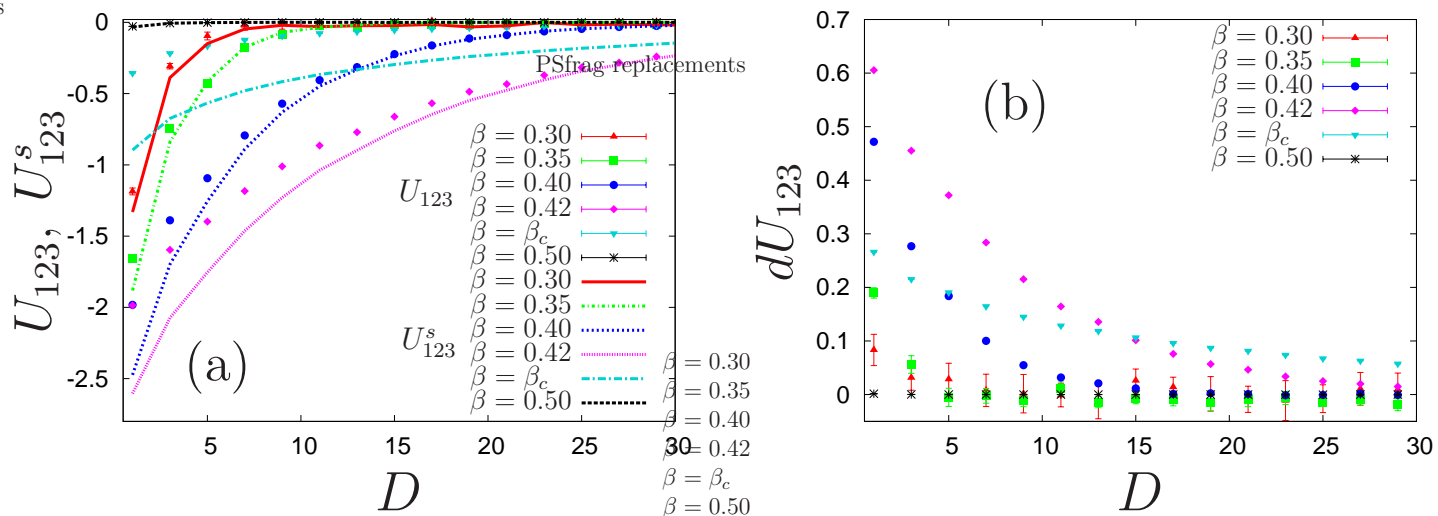

FIG. 5: (Color online) (a) Multiparticle CC interaction potential $U_{123}$ (symbols) between three disks located in vertices of the equilateral triangle as a function of the side length $D$ for various values of the inverse temperature $\beta=0.3,0.35,0.4,0.42, \beta_{c}, 0.5$ in comparison with the sum of three pair potentials $U_{123}^{s}=U_{12}+U_{13}+U_{23}$ (lines); (b) Excess non-additive contribution to CC interaction potential $d U_{123}=U_{123}-U_{123}^{s}$ for $\beta=0.3,0.35,0.4,0.42, \beta_{c}, 0.5$.

temperature $\beta$ for different separations between disks $D=1,3,5,7,11,19$ by symbols. In the same figure we plot the sum of pair potentials $U_{123}^{s}$ by lines. The difference between $U_{123}$ 
and $U_{123}^{s}$ demonstrates us the non-additive nature of multiparticle CC interactions. Let us underline, that for the fixed value of $\beta$ all quantities: $\left\langle 2^{-c_{123}(D)}\right\rangle_{\beta},\left\langle 2^{-c_{12}(D)}\right\rangle_{\beta},\left\langle 2^{-c_{13}(D)}\right\rangle_{\beta}$, $\left\langle 2^{-c_{23}(D)}\right\rangle_{\beta}$ for all set of separations $D$ are computed during the single simulation of the bulk system! It means, that we should not perform separate simulation for each value of $D$. In Fig. 4(b) we plot the non-additive difference $d U_{123}=U_{123}-U_{123}^{s}$ as a function of $\beta$ for the same systems. The non-additive part of interactions is positive. It means, that the presence of the third particle decreases the interaction between pair of particles. In Fig. 4(b) we see, that the non-additive part of three-particle interactions has the maximum approximately at the same point, as the minimum of two-particle interaction potential. All these potentials have wide tails to the high-temperature region, while interactions in low-temperature region decreases very fast as we separates from the critical point. This behavior qualitatively coincide with results for MF system [29, 30] and with experimental results [15] for similar geometries.

In Figs. 5(a) and (b) we plot multiparticle interaction potential and its excess non-additive contribution as functions of the separation $D$ for various values of the inverse temperature $\beta$, respectively. Such presentation is more convenient for experimentators, who typically measure the potential as a function of separation for the given temperature [7]. The nonadditivity of three-particle interaction for 2D system at the critical point has been previously studied in Ref. [43]. In this article the information about interaction potentials is extracted from particle distribution functions. Contrary to this approach, our method provides the possibility to compute the interaction potential directly, simultaneously for several values of separations $D$.

\section{CONCLUSION}

In the present paper we express the free energy change for the insertion of objects into a critical system in terms of percolation clusters intersecting the volume of inserting objects. The numerical algorithm for CC interactions which is based on counting of the number of percolation clusters is proposed. The algorithm provides explicit expression for the Casimir potential without numerical integration. This algorithm is numerically verified for the computation of CC force potential between two disks for 2D Ising model. The proposed method is also applied for the studying of multiparticle interactions in a critical media and express 
the non-additive part of the interaction potential in terms of percolation clusters. Obtained expression gives us the qualitative information about the sign of the non-additive contribution to multiparticle interaction while the numerical realization of the proposed algorithm let us to perform quantitative computation of the interaction potential. Results for the temperature dependence of the non-additive part of three-particle interaction for 2D system are presented first time.

[1] M.E. Fisher and P.G. de Gennes, C.R. Acad. Sci. Paris Ser. B 287, 207 (1978).

[2] M. Krech, Casimir Effect in Critical Systems (World Scientific, Singapore, 1994).

[3] J.G. Brankov, D.M. Dantchev, and N.S. Tonchev, The Theory of Critical Phenomena in Finite-Size Systems - Scaling and Quantum Effects (World Scientific, Singapore, 2000).

[4] A. Gambassi, J. Phys.: Conf. Ser. 161, 012037 (2009).

[5] M. Fukuto, Y.F. Yano, and P.S. Pershan, Phys. Rev. Lett. 94, 135702 (2005).

[6] D. Beysens and D. Estève, Phys. Rev. Lett. 54, 2123 (1985).

[7] C. Hertlein, L. Helden, A. Gambassi, S. Dietrich, and C. Bechinger, Nature 451, 172 (2008).

[8] A. Gambassi, A. Maciołek, C. Hertlein, U. Nellen, L. Helden, C. Bechinger, and S. Dietrich, Phys. Rev. E 80, 061143 (2009).

[9] S. Buzzaccaro, J. Colombo, A. Parola, and R. Piazza, Phys. Rev. Lett. 105, 198301 (2010).

[10] R. Piazza, S. Buzzaccaro, A. Parola, and J. Colombo, J. Phys.: Condens. Matter 23, 194114 (2011).

[11] S.J. Veen, O. Antoniuk, B. Weber, M.A.C. Potenza, S. Mazzoni, P. Schall, and G.H. Wegdam, Phys. Rev. Lett. 109, 248302 (2012).

[12] V.D. Nguyen, S. Faber, Z. Hu, G.H. Wegdam, and P. Schall, Nature Comm. 4, 1584 (2013).

[13] M.T. Dang, A.V. Verde, V.D. Nguyen, P.G. Bolhuis, and P. Schall, J. Chem. Phys. 139, $094903(2013)$.

[14] V.D. Nguyen, M.T. Dang, T.A. Nguyen, and P. Schall, J. Phys.: Condens. Matter 28, 043001 (2016).

[15] S. Paladugu, A. Callegari, Y. Tuna, L. Barth, S. Dietrich, A. Gambassi, and G. Volpe, Nature Comm. 7, 11403 (2016).

[16] O. Vasilyev, A. Gambassi, A. Maciołek, and S. Dietrich, EPL 80, 60009 (2007). 
[17] O. Vasilyev, A. Gambassi, A. Maciołek, and S. Dietrich, Phys. Rev. E 79, 041142 (2009).

[18] M. Hasenbusch, Phys. Rev. B 82, 104425 (2010).

[19] O. Vasilyev, A. Maciołek, S. Dietrich Phys. Rev. E 84, 041605 (2011).

[20] O. Vasilyev and S. Dietrich, EPL 104, 60002 (2013).

[21] D.L. Cardozo, H. Jacquin, P.C.W. Holdsworth, Phys. Rev. B 90, 184413 (2014).

[22] M. Hasenbusch, Phys. Rev. E 87, 022130 (2013).

[23] O.A. Vasilyev, Phys. Rev. E 90, 012138 (2014).

[24] O. Vasilyev, A. Maciołek, Journal of Non-Crystalline Solids 407, 376 (2015).

[25] E. Eisenriegler and U. Ritschel, Phys. Rev. B 51, 13707 (1995).

[26] F. Schlesener, A. Hanke, and S. Dietrich, J. Stat. Phys. 110, 981 (2003).

[27] S. Kondrat, L. Harnau, and S. Dietrich, J. Chem. Phys. 131, 234902 (2009).

[28] T.F. Mohry, S. Kondrat, A. Maciołek, and S. Dietrich, Soft Matter 10, 5510 (2014).

[29] T.G. Mattos, L. Harnau, and S. Dietrich, J. Chem. Phys. 138, 074704 (2013).

[30] T.G. Mattos, L. Harnau, and S. Dietrich, Phys. Rev. E 91, 042304 (2015).

[31] S.L. Veatch, P. Cicuta, P. Sengupta, A. Honerkamp-Smith, D. Holowka, and B. Baird, ACS Chem. Biol. 3, 287 (2008).

[32] B. Machta, S. Papanikolaou, J.P. Sethna, and S.L. Veatch, Biophys. J. 100, 1668 (2011).

[33] E. Gray, J. Karslake, B.B. Machta, and S.L. Veatch, Biophys. J. 105, 2751 (2013).

[34] A. Drzewiński, A. Maciołek, and R. Evans, Phys. Rev. Lett. 85, 3079 (2000).

[35] A. Maciołek, A. Drzewiński, and R. Evans Phys. Rev. E 64, 056137 (2001).

[36] D.B. Abraham and A. Maciołek, Phys. Rev. Lett. 105, 055701 (2010).

[37] D.B. Abraham and A. Maciołek, 101, 20006 (2013).

[38] M. Zubaszewska, A. Maciołek, and A. Drzewiński, Phys. Rev. E 88, 052129 (2013).

[39] H. Hobrecht and A. Hucht, EPL 106, 56005 (2014).

[40] B.B. Machta, S.L. Veatch, and J.P. Sethna, Phys. Rev. Lett. 109, 138101 (1012).

[41] O.A. Vasilyev, E. Eisenriegler, and S. Dietrich, Phys. Rev. E 88, 012137 (2013).

[42] J.R. Edison, N. Tasios, S. Belli, R. Evans, R. van Roij, and M. Dijkstra, Phys. Rev. Lett. 114, $038301(2015)$.

[43] H. Hobrecht and A. Hucht, Phys. Rev. E 92, 042315 (2015).

[44] E. Eisenriegler, T.W. Burkhardt, Phys. Rev. E 94, 032130 (2016).

[45] P.W. Kasteleyn and C.M. Fortuin, Physica 57, 536 (1972). 
[46] J.W. Essam, Rep. Prog. Phys. 43, 833 (1980).

[47] D. Stauffer, A. Aharony, Introduction to Percolation Theory, (second ed., Taylor and Francis, London, 1994).

[48] A.A. Saberi, Physics Reports 578, 1 (2015).

[49] A. Coniglio and W. Klein, J. Phys. A 13, 2775 (1980).

[50] M.O. De Meo, D.W. Heermann, and K. Binder, J. Stat. Phys. 60, 585 (1990).

[51] R.H. Swendsen and J.S. Wang, Phys. Rev. Lett. 58, 58 (1986).

[52] D.P. Landau and K. Binder, A Guide to Monte Carlo Simulations in Statistical Physics (Cambridge University Press, London, 2005).

[53] N. Metropolis and S. Ulam, J. Amer. Stat. Assoc. 44, 335 (1949).

[54] O. Vasilyev, in Order, Disorder and Criticality: Advanced Problems of Phase Transition Theory Vol. 4, edited by Y. Holovatch (World Scientifc, Singapore, 2015) p. 55. 